\title{
Niveles de plagio y uso de normas para presentación de trabajos en un programa de pregrado en la ciudad de Barranquilla
}

\author{
Levels of plagiarism and use of standards for presentation of work in an undergraduate
} program in the city of Barranquilla

DOI: https://doi.org/10.21803/penamer.14.27.318

Isaac Nieto Mendoza

http://orcid.org/0000-0001-5302-6931

\section{¿Cómo citar este artículo?}

Nieto, I. (2021). Niveles de plagio y uso de normas para presentación de trabajos en un programa de pregrado en la ciudad de Barranquilla. Pensamiento Americano, 14(27), 137-144

DOI: https://doi.org/10.21803/penamer.14.27.318

\section{Resumen}

Introducción: Se realizó un estudio de investigación relacionado con los trabajos de grado aceptados para optar por el título académico en la universidad estudiada de la ciudad Barranquilla, Colombia entre los años 2015 y 2020. Objetivo, Método y/o Metodología: Esta investigación tuvo como objetivo caracterizar la producción académica de un programa de la ciudad de Barranquilla, haciendo énfasis en aspectos como la modalidad de grado, metodología de investigación, uso de normas para presentación de trabajos establecidas por la American Psychological Association (APA) por medio de un diseño metodológico cuantitativo y tipo descriptivo-documental. Resultados y conclusiones: De las 50 tesis evaluadas, el $64 \%$ fueron monografías, el $86 \%$ de los trabajos no fue presentado bajo las normas APA y el $44 \%$ presenta niveles de plagio entre el 10 y $15 \%$. Estos resultados dan cuenta de la falta de rigor en la revisión de los proyectos de grado, infringiendo los criterios establecidos en el reglamento de trabajo de grado del programa estudiado en lo que respecta a su estructura, niveles de plagio y uso de normas APA.

Palabras clave: Trabajos de grado; Normas APA; Plagio.

\begin{abstract}
Introduction: A research study was conducted related to the accepted undergraduate work to opt for the academic degree at the university studied in the city of Barranquilla, Colombia between 2015 and 2020. Objective, Method and/or methodology: This research aimed to characterize the academic production of a program in the city of Barranquilla, emphasizing aspects such as the modality of degree, research methodology, use of standards for submission of papers established by the American Psychological Association (APA) by means of a quantitative methodological design and descriptive-documentary type. Results and conclusions: Of the $\mathbf{5 0}$ theses evaluated, $64 \%$ were monographs, $86 \%$ of the works were not presented under APA standards and $44 \%$ presented plagiarism levels between 10 and $15 \%$. These results show the lack of rigour in the review of the degree projects, in violation of the criteria established in the grade work regulations of the studied program in terms of their structure, levels of plagiarism and use of APA standards.
\end{abstract}

Keywords: Degree work; APA standards; Plagiarism. 


\section{Resumo}

a

Palavras-chave: a 


\section{Introducción}

La revisión y análisis de la producción académica en los entornos universitarios, constituye un aporte hacia la búsqueda de mejorar los procesos de generación de conocimiento, para la obtención de mayor calidad e impacto en dichos contextos (Cortés-Trujillo, 2015; Escorcia-Otálora, 2008; Pérez-Jiménez et al., 2020; Pérez-Saavedra et al., 2008; Reguera, 2008; Roig-Villa et al., 2016).

Es evidente, según Pérez-Jiménez et al. (2020) en la realización de trabajos de grado, el desconocimiento sobre la metodología de investigación a seleccionar, debido a los sesgos existentes en materia de enseñanza de los distintos diseños de investigación existentes. Es por eso que este autor manifiesta una problemática imperante, especialmente en ciencias humanas sobre cómo explicar el proceder metodológico de su proyecto de grado, por lo cual, optan por omitirlo y resulta difícil una lectura certera del mismo.

También, de acuerdo con Cisneros- Estupiñán (2012), una de las mayores dificultades en la producción de trabajos de grado en los entornos universitarios latinoamericanos está asociada con la reproducción de conocimientos de autores especializados, debido a la incapacidad de interpretar, codificar y expresar lo entendido a través de un determinado estudio a expertos en la materia que se aborda. Esto se debe, además, según Venegas et al, (2016), al desconocimiento de las normas establecidas por las universidades para la presentación de trabajos, concordando estos estudios en que las normas de mayor aceptación han sido las normas establecidas por la American Psychological Association (APA).

Las falencias en cuanto al análisis y comprensión de textos y el desconocimiento de la forma adecuada de referenciación, ha conllevado al estudiante de pregrado a la exposición de ideas de autores sin tener en cuenta el respeto por la propiedad intelectual al igual que desconocer - dentro del texto- la fuente de la que fue obtenida y referenciada debidamente; esta falencia, según Hernández-Islas (2016), es muy común en la presentación de trabajos por parte de universitarios, apropiándose de la propiedad intelectual ajena y asumiéndola como suya, de manera involuntaria y, en la mayoría de casos, voluntaria.

Por otra parte, el uso indebido de propiedadintelectual en entornos universitarios está relacionado con el desconocimiento de las normativas para presentación de trabajos, además que muchos docentes desconocen los softwares para identificar el plagio (Merelles, 2017; Murillo, 2011; Ronda-Pérez, et al., 2016; Torres et al, 2013; Timal y Sánchez, 2017). Por tanto, las universidades deberán preocuparse por generar estrategias de alto impacto que induzcan a la profesionalización de la investigación y la educación. En Colombia, según la Corte Suprema de Justicia de Colombia (2010, citada en Sanabria, 2014), el plagio puede ser clasificado de la siguiente manera:

(...) uno, cuando el actor se atribuye la obra de otro, tal y como ha sido creada por el autor; y dos, cuando el actor se atribuye la obra de otro, no reproduciéndola idénticamente, sino imitándola en sus extremos esenciales (...) (p. 88).

Los aspectos antes mencionados resultan un punto de partida para realizar esta investigación, la cual tiene como objetivo caracterizar la producción académica de pregrado en el programa de la Universidad estudiada en los últimos 5 años, teniendo en cuenta aspectos básicos relacionados con la corriente filosófica, la modalidad o tipología de trabajo de grado y aspectos de profundidad como la metodología de la investigación, el uso de normas para presentación de trabajos establecidas por la American Psychological Association (APA), la cantidad de referencias bibliográficas y los niveles de plagio. Esto se hace con el fin de conocer cómo se ha llevado a cabo la producción de proyectos de grado en el programa de la universidad estudiada, a fin de servir a los tomadores de decisiones de la Facultad donde se encuentra adscrita la misma para el fortalecimiento de la cátedra Trabajo de Grado. 


\section{METODOLOGÍA}

\subsection{Diseño}

Estudio descriptivo y enfoque cuantitativo, teniendo en cuenta 50 trabajos de grado del programa de un programa de una universidad de la ciudad de Barranquilla, Colombia, realizados entre los años 2015 y 2020 y que reposan en la biblioteca del contexto universitario mencionado, por lo que son de acceso abierto; asimismo, se realizó una revisión documental de los textos entre el mes de marzo de 2019 y abril del 2020, excluyéndose aquellas tesis de otras instituciones afiliadas a la universidad.

\subsection{Instrumentos}

Para la recolección de la información, se siguieron los lineamientos establecidos en el formato de evaluación para trabajos de grado de la universidad estudiada y el Reglamento de Trabajo de Grado del programa (Universidad del Atlántico, 2017). Los criterios a tener en cuenta en la revisión de los trabajos de grado fueron los siguientes:

Número de tesis por año: se identificaron las tesis y artículo que se encontraban entre el rango de años 2015-2020 que fueron aprobadas por el Comité de Consejo Académico del programa estudiado, y que reposaban en la biblioteca de la misma con acceso abierto.

Tipología o modalidad de trabajo de grado: son las opciones de trabajo de grado del programa, aceptados como requisito para finalización de la carrera y son, monografía, ensayo, revisión bibliográfica, traducción y artículo publicado y aprobado para publicación.

Metodología de la investigación: el diseño metodológico, enfoque y fases de la investigación seleccionadas para llevar a cabo el trabajo de grado.

Uso de normas APA: se tiene en cuenta el uso de la normativa APA de la edición correspondiente a los años de los trabajos de grado revisados; los aspectos evaluados son, formato de las referencias, estructura de los párrafos y citación dentro del texto.

Número de referencias bibliográficas: cantidad de fuentes presentadas em la bibliografía.

Nivel de plagio: porcentajes de plagio obtenidos a través del programa Turnitin ${ }^{\circledR}$.

La lista de análisis o formulario fue puesta en consideración ante comité de expertos de la universidad estudiada y aprobado para su aplicación el día 14 de febrero de 2019.

\subsection{Análisis de la información}

El análisis estadístico de los resultados se efectuó por medio del programa IBM SPSS V. 23 para Windows 10. La interpretación de los datos se hizo por medio del análisis univariado, midiendo las variables en escala nominal y describiendo los datos mediante frecuencia y porcentaje, mostrando la información en tablas y figuras, presentando las variables numéricas a través de promedios, valor máximo y valor mínimo.

\subsection{Consideraciones éticas}

Esta investigación fue puesta en consideración ante la coordinación del programa estudiado en el año 2020; asimismo, teniendo en cuenta la calidad de acceso abierto de los trabajos de grado, no se requirió de permiso alguno por parte del comité de ética de la universidad en que se realizó el estudio. Se tuvieron en cuenta los aspectos legales establecidos en el Decreto 1377 (2013), referentes a la protección de la identidad y el tratamiento de datos.

\section{RESULTADOS}

De acuerdo con la Figura 1, de las 50 tesis producidas entre los años 2015 y 2020, 14 de ellas corresponden al año 2017 y la menor producción al 2020 con 2 tesis; se evidencia que la mayor producción de proyectos de grado en el programa se presentó desde el año 2016 al 2018, disminuyendo en el año 2019 hasta 2020. 


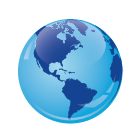

Figura 1

Número de trabajos de grado en los últimos 5 años.

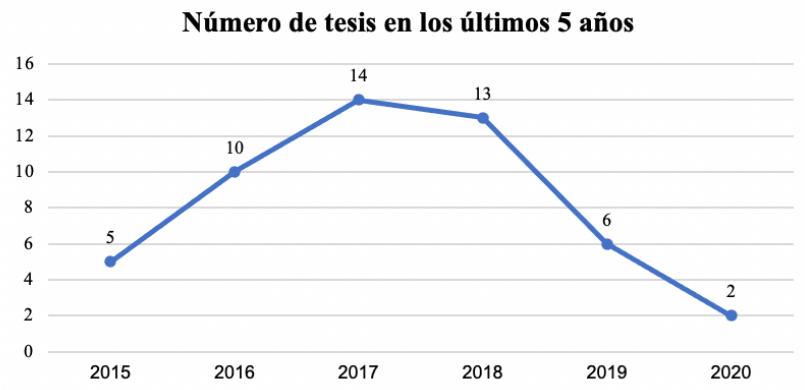

En la Tabla 1, con relación a la tipología de proyectos de grado se han realizado mayormente monografías (64\%) y en menor proporción artículos científicos (2\%); en lo que corresponde a la metodología de la investigación, los estudiantes en el desarrollo de su trabajo, no especifican el diseño metodológico ni presentan las fases de abordaje del estudio (76\%). Con respecto al uso de las normas establecidas por la American Psychological Association para el desarrollo de trabajos, son incumplidas por el $86 \%$ de los estudiantes del programa b. Además, en cuanto al número de referencias bibliográficas utilizadas en la justificación de su trabajo de grado, se evidencia un uso de menos de 25 referencias bibliográficas (74\%) y niveles de plagio - en su mayoría- entre el 10 y el 15 por ciento $(44 \%)$.

\section{DISCUSIÓN}

La prevalencia de modalidad o tipología de trabajo de grado monografía (64\%), resulta un dato interesante, ya que, al hacer un estudio minucioso de los proyectos de grado y, teniendo en cuenta lo establecido en el Reglamento de Trabajo de Grado del Programa (Universidad del Atlántico, 2017), con respecto a la modalidad en mención, su estructura de adapta a los criterios establecidos para ensayo, puesto que la totalidad de las monografías revisadas no presenta apartados como "planteamiento del problema, justificación, objetivos, marco histórico, marco teórico, estado del arte, metodología, discusión o resultados y conclusiones y propuestas" (Universidad del Atlántico, 2017, pp. 2-3), que son propiedades que debe contener una monografía.

De los resultados obtenidos a través de la investiga-
Tabla 1.

Criterios generales de análisis para los trabajos de grado.

\begin{tabular}{crr}
\hline Criterios de análisis & n & $\%$ \\
\hline Tipología de Trabajo de Grado & & \\
Monografía & 32 & $64 \%$ \\
Articulo Científico & 1 & $2 \%$ \\
Revisión bibliográfica & 7 & $14 \%$ \\
No se describe & 10 & $20 \%$ \\
Metodología de la investigación & & \\
Investigación cualitativa & 12 & $24 \%$ \\
No se especifica & 38 & $76 \%$ \\
Uso de Normas APA & & \\
Si & 7 & $14 \%$ \\
No & 43 & $86 \%$ \\
Número de referencias bibliográficas & \\
$<25$ & 37 & $74 \%$ \\
$25-50$ & 6 & $12 \%$ \\
$>50-75$ & 5 & $10 \%$ \\
$>75$ & 2 & $4 \%$ \\
$<5 \%$ & 8 & $16 \%$ \\
Nivel de plagio & 6 & $12 \%$ \\
$<\%-10 \%$ & 22 & $44 \%$ \\
$10 \%-15 \%$ & 14 & $28 \%$ \\
$<15 \%$ & &
\end{tabular}

ción abordada, es preciso resaltar la tendencia de los trabajos de grado realizados en el programa en los últimos 5 años hacia niveles de plagio considerables, evidenciando la asunción por parte de los autores de conceptos ajenos como producción intelectual propia, lo cual concuerda con lo manifestado por Cisneros- Estupiñán (2012), considerando común la reproducción por parte de universitarios latinoamericanos de ideas que son propiedad de autores especializados; asimismo, concuerda con lo establecido por Hernández-Islas (2016), quien concibe las prácticas de plagio como comunes en los contextos universitarios, haciendo uso de la propiedad intelectual de otros y presentándola como suya.

A pesar de encontrarse la mayoría de trabajos de grado en un rango aceptable de plagio (10\% - 15\%) de acuerdo como lo establece Valderrama (2016), es posible apreciar la prevalencia de trabajos de grado aceptados con niveles representativos de plagio superiores al 15\%, lo cual discrepa con lo establecido en el Reglamento de Trabajo de Grado del Programa (Universidad 
del Atlántico, 2017), donde se indica que, la presencia de plagio en un trabajo de grado deberá ser notificada por los evaluadores asignados para la revisión de dicho trabajo y que, de ser notificado el uso de propiedad intelectual "total o parcial, voluntaria o involuntaria” (p.7) será pretexto para la cancelación o establecer sanciones respectivas del trabajo presentado.

Lo anterior da cuenta de la falta de rigurosidad en la revisión de los trabajos de grado realizados en el programa, puesto que es deber de los docentes evaluadores del trabajo de grado asignado, informar con pruebas las faltas del estudiante relacionadas con el uso de la propiedad intelectual ajena; no obstante, la prevalencia de los modelos tradicionalistas de revisión de proyectos de grado, han conllevado a la reproducción de tesis de grado con alto contenido de plagio, desconociendo el uso de softwares especializados para la identificación este (Ronda-Pérez, et al., 2016; Murillo, 2011; Torres et al., 2013; Merelles, 2017), lo cual se ve reflejado en el programa en mención, puesto que, a pesar de disponer de softwares anti-plagio como Turnitin, no han recibido la capacitación respectiva sobre su uso.

Otro hallazgo importante fue el referente al uso de las normas para presentación de trabajos (APA), donde el $86 \%$ de los trabajos realizados y aprobados entre 2015 y 2020 no aplican debidamente dicha norma, lo cual discrepa de lo establecido en el Reglamento de Trabajo de Grado del Programa (Universidad del Atlántico, 2017), donde se especifica que, aquellos trabajos de grado que no sean presentados según las Normas APA, no serán aceptados; esto concuerda con lo afirmado por Venegas et al. (2016) y Hernández-Islas (2016), quienes atribuyen al alto nivel de plagio en los trabajos de grado por parte de los universitarios al desconocimiento y poca instrucción en el uso de normas APA.

Si bien, el plagio puede ser involuntario (Sanabria, 2014), y relacionarse con la falta de rigurosidad en el uso de las normas para presentación de trabajos (Venegas, et al., 2016; Hernández-Islas, 2016), el Reglamento de Trabajo de Grado del Programa (Universidad del Atlántico, 2017), "no es argumento el desconocimiento de la aplicación de la norma, pues es deber del estudiante revisar la parte metodológica del trabajo” (p. 7), lo cual es distinto a lo que se hace evidente en los proyectos de grado evaluados entre los años 2015 y 2020 , ya que estos documentos cuentan con el aval docente a pesar de violar la cláusula mencionada.

Es de resaltar también el porcentaje alto de trabajos de grado que presentan un número menor a 25 referencias (74\%), lo que permite aseverar, al revisar de manera exhaustiva los documentos, la presencia de bibliografía incompleta, es decir, referencias citadas dentro del texto pero que no son mencionadas en la bibliografía; esto puede estar asociado al desconocimiento y falta de uso de las normas para presentación de trabajos APA.

\section{CONCLUSIONES}

Tras los resultados obtenidos, es posible afirmar las grandes dificultades que se hacen evidentes en materia de desarrollo de trabajos de grado en el programa estudiado, donde se aprecia falta de rigor por parte de los evaluadores de trabajos de grado, especialmente en materia de distinguir propiedad intelectual del estudiante y plagio, infringiendo lo establecido en los reglamentos institucionales referente a la revisión exhaustiva y manifestación de inconsistencias para el establecimiento de sanciones.

Es preciso que los académicos velen por la producción académica de calidad e inducir esta cultura investigativa en los universitarios, induciéndolos a seguir lineamientos preestablecidos sobre la presentación de trabajos de grado, el uso de normas APA y la búsqueda de bibliografía en base de datos, a fin de alimentar sus proyectos con referentes teóricos acordes con su temática; por tanto, es pertinente que se dicten cátedras relacionadas con el uso de las normas para presentación de trabajos y metodología de la investigación, a fin de que los universitarios tengan los conocimientos sobre cómo realizar un proyecto de grado.

Con lo anterior, resulta una necesidad fundamental la socialización de los lineamientos establecidos en el reglamento para presentación de trabajo de grado, puesto que, el estudiante, al desconocer la estructura,

Pensamiento Americano Vol. 14 - No. 27 - p.p. 137- 144 • 2021 • Enero-Junio • Corporación Universitaria Americana • Barranquilla, Colombia • ISSN-e: 2745-1402 
opta por realizar su proyecto bajo ninguna estructura, lo que dificulta su revisión por parte de los evaluadores e implica una demora en la culminación del proyecto de grado; esto se ve reflejado en la baja producción de trabajos de grado que se ha presentado en los últimos 5 años en comparación con otros programas académicos de la universidad estudiada.

Finalmente, es importante que los docentes del programa y, en general, los académicos de la facultad a la que está adscrito el mismo, se capaciten en el uso de softwares para la detección de plagio, a fin de realizar revisiones preliminares del trabajo de grado e inducir al estudiante a realizar los correctivos correspondientes relacionados con el respeto de la propiedad intelectual ajena, además de agilizar y facilitar la revisión de los mismos.

\section{LIMITACIONES}

La investigación hubiese tenido un mayor impacto de haber abarcado los estudios realizados desde el año 2010 hasta el 2020, lo cual no fue posible ya que muchos de los trabajos de años anteriores al 2015, no reposan en la biblioteca de la universidad estudiada, lo cual dificultó su acceso.

\section{Referencias}

Cisneros-Estupiñán, M. (2012). ¿Cómo elaborar trabajos de grado? (Segunda ed.). ECOE Ediciones.

Cortés-Trujillo, J. (2015). Dificultades de la escritura de informes de investigación formativa en la educación superior en facultades de ingeniería. Revista Polisemia, 8(14), 12-23. doi:https://doi.org/10.26620/uniminuto.polisemia.8.14.2012.12-23

Escorcia-Otálora, T. (2008). El análisis bibliométrico como herramienta para el seguimiento de publicaciones científicas, tesis y trabajos de grado. (Tesis de Pregrado, Pontificia Universidad Javeriana). Archivo digital. https://www.javeriana.edu.co/biblos/tesis/ciencias/tesis209.pdf

Hernández-Islas, M. (2016). El plagio académico en la investigación cinetífica: consideraciones desde la óptica del investigador de alto nivel. Perfiles Educativos, 38(153), 120-135.

Merelles, M. (2017). El uso de recursos abiertos como apoyo en la implementación de las Normas APA. (Tesis de Pregrado. Tecnológico de Monterrey). Repositorio Institucional del Tecnológico de Monterrey. https://repositorio.tec.mx/ handle/11285/622475

Murillo, J. (2011). Recomendaciones para escribir un ensayo: Normas APA. Colegio de Estudios Superiores de Administración. https://repository.cesa.edu.co/bitstream/handle/10726/301/Normas\%20APA_web.pdf?sequence=1\&isAllowed $=y$

Pérez-Jiménez, C., Cruz, O., García, G., Ocaña, J. \& Hernández, S. (2020). Consideraciones en la enseñanza de la metodología de la investigación en las Ciencias Humanas. Revista Iberoamericana de Producción Académica y gestión Educativa, $7(13), 1-17$.

Pérez-Saavedra, V., Zárate, M., Gonzáles, R. \& Ampuero, E. (2008). Caracterización de las tesis de pregrado de la facutad de Enfermería de la Universidad Peruana Cayetano Heredia. Revista Enfermería Herediana, 1(1), 48-56. 
pública de Colombia. Diario Oficial: 48.834

Reguera, A. (2008). Metodología de la investigación lingüistica: prácticas de escritura. Editorial Brujas.

Roig-Villa, R., Blasco, J., Lledó, A., \& Pellín, N. (2016). Investigación e Innovación Educativa en Docencia Universitaria. Retos, Propuestas y Acciones. Universidad de Alicante. Instituto de Ciencias de la Educación

Ronda-Pérez, E., Seguí, M., Cayuela, A., Tauste, A. \& Esteve, J. (2016). Plagio académico en el alumnado académico de máster en la universidad de Alicante. Universidad de Alicante. https://rua.ua.es/dspace/bitstream/10045/60520/1/ Investigacion-e-Innovacion-Educativa-en-Docencia-Universitaria_105.pdf

Sanabria, L. (2014). Conceptualización jurídica del plagio en Colombia. Revista Colombiana de Cirugía, 29(2), 88-97.

Timal, S. \& Sánchez, F. (2017). El plagio en el contexto del derecho de autor. Tla-melaua, 11(42), 48-66.

Torres, S., González, A. \& Vavilona, I. (2013). La cita y referencia bibliográfica: guía basada en las Normas APA (Tercera ed.). Biblioteca UCES. https://www.revistaespacios.com/ citasapa.pdf

Universidad del Atlántico. (2017). Reglamento de Trabajo de Grado del Programa. https://uniatlantico.edu.co/uatlantico/sites/default/files/docencia/facultades/pdf/ciencias- humanas/filosofia/NUEVO\%20REGLAMENTO\%20TG\%20 PROGRAMA \%20DE\%20FILOSOFI\%CC\%81A.pdf

Valderrama, J. (2016). ¿Cuánto debo plagiar para no ser acusado de plagiador? Formación Universitaria, 9(4), 1-2.

Venegas, R., Zamora, S. \& Galdames, A. (2016). Hacia un modelo retórico-discrusivo del macrogénero Trabajo Final de Grado en Licenciatura. Revista Signos, 49(1), 247-279.

2021, Vol. 14(27) 137-144. (CThe Author(s) 2021 